Check for updates

Cite this: RSC Adv., 2017, 7, 45682

Received 25th May 2017

Accepted 6th September 2017

DOI: $10.1039 / \mathrm{c} 7 \mathrm{ra0} 5875 f$

rsc.li/rsc-advances

\section{Giant Pockels effect of polar organic solvents and water in the electric double layer on a transparent electrode}

\author{
Hironori Kanemaru, ${ }^{a}$ Shunpei Yukita, ${ }^{a}$ Hajime Namiki, ${ }^{a}$ Yugo Nosaka, ${ }^{a}$ \\ Takayoshi Kobayashibc and Eiji Tokunaga (D)*ad
}

The Pockels effect of polar organic solvents and water within the electric double layer (EDL) on an indiumtin-oxide (ITO) electrode is studied to find that water has the largest Pockels coefficient (230 pm V $^{-1}$ ), followed in order by methanol (200 pm $\mathrm{V}^{-1}$ ), ethanol $\left(84 \mathrm{pm} \mathrm{V}^{-1}\right.$ ), and dimethyl sulfoxide (DMSO) (20 $\mathrm{pm} \mathrm{V}^{-1}$ ). Electrolyte solutions of water and methanol have nearly the same magnitude of Pockels coefficient, while ethanol and DMSO solutions exhibit two and ten times smaller Pockels coefficients than the methanol solution, respectively. The Pockels coefficient scales well with the hydrogen-bond strength (or average cluster size) divided by the solvent viscosity. This suggests that hydrogen bonding and viscosity play crucial roles in the mechanism of the Pockels effect of these liquids.

\section{Introduction}

The linear electro-optic (EO) effect, or Pockels effect, exists only in crystals that do not possess inversion symmetry. ${ }^{1}$ Therefore it is usually ruled out to use bulk liquid, where constituent molecules are randomly oriented to keep macroscopic centrosymmetry, as an EO device. Recently, the EO effect of water within the electric double layer (EDL) at the electrode-solution interface ${ }^{2,3}$ was reported to have a Pockels constant one order of magnitude larger than that in typical electro-optic crystals such as $\mathrm{LiNbO}_{3} \cdot{ }^{1,4,5}$ The thickness of the EDL defined by the DebyeHückel length ${ }^{2}$ is estimated to be of the nanoscale-order. There is also an intriguing report of the Pockels effect even in a bulk water region between the electrodes. ${ }^{6,7}$ Recently, the Pockels effect was observed at the air-water interface, too. ${ }^{8}$ Then, there arise natural questions: whether water is a special liquid in that it possesses a large Pockels coefficient? Do other liquids such as organic solvents also have large Pockels coefficients?

The principle for the Pockels effect of water to occur is the initially induced broken centrosymmetry due to the presence of the electrode surface even without an applied electric field, but a microscopic physical mechanism to predict the size of the Pockels effect is yet to be clarified. From the theoretical side,

aDepartment of Physics, Faculty of Science, Tokyo University of Science, 1-3 Kagurazaka, Shinjuku-ku, Tokyo 162-8601, Japan. E-mail: eiji@rs.kagu.tus.ac.jp

${ }^{b}$ Advanced Ultrafast Laser Research Center, Brain Science Inspired Life Support Research Center, The University of Electro-Communications, 1-5-1 Chofugaoka, Chofu, Tokyo 182-8585, Japan

'Advanced Ultrafast Laser Research Center, Department of Electrophysics, National Chiao-Tung University, 1001 Ta Hsinchu Rd., Hsinchu 300, Taiwan

${ }^{d}$ Research Center for Water Frontier Science and Technology, Tokyo University of Science, 1-3 Kagurazaka, Shinjuku-ku, Tokyo 162-8601, Japan this is probably because the electronic structure needs to be modelled precisely in order to predict the dynamic polarizability of the interfacial water, ${ }^{9}$ but it is difficult to conduct $a b$ initio calculations of both the molecular and electronic structures of electrolyte dissolved liquid water at the liquid-solid interface in the presence of an electric field. ${ }^{10}$ Recent advances in computational methodology for simulating nonlinear optical properties realize rigorous calculation of the dynamic electronic hyper-polarizability of crystalline systems and surfaces to reproduce experimental data. ${ }^{11}$ Thus there is an expectation that the Pockels effect of water is a potentially solvable problem computationally. From the experimental side, there is insufficient information about which properties of water play crucial roles. The electrode surface can induce orientational ordering of water ${ }^{12}$ or the interaction of water molecules with the atoms of the electrode surface. ${ }^{3,13,16}$ Orientational ordering of water molecules in the EDL is a possible mechanism, but the principal dynamic polarizability of the water molecules has been reported to be nearly isotropic both experimentally ${ }^{14}$ and theoretically. ${ }^{15}$ Thus, a hydrogen-bond network of water or the interaction of water molecules with the surface atoms of the electrode were suggested to be mechanisms. ${ }^{4,5}$ It is therefore interesting to investigate whether the large Pockels effect is specific to liquid water, or is common to other liquids such as organic solvents.

Water is a special liquid because of its strong hydrogen bonding network. ${ }^{17-21}$ Thus, the relevance of the hydrogen bond $^{22}$ to the Pockels effect is of great interest. In this paper therefore, we have studied the Pockels effect of three protonic solvents (water, methanol, and ethanol) and one aprotonic solvent (dimethyl sulfoxide, DMSO). Here, polar solvents are chosen because electrolytes need to be dissolved to form the 
EDL on the electrode for the Pockels effect to occur, as evidenced by the fact that the magnitude of the Pockels effect is proportional to the square root of the ionic strength. ${ }^{5}$ We have found that methanol and ethanol show a Pockels effect comparable to that for water while DMSO shows a much smaller effect. This is the first observation of the Pockels effect of organic solvents to the best of our knowledge.

The EDL provides a range of various electrochemical reactions at the solid-solution interface. Electrochemistry in nonaqueous solutions ${ }^{23,24}$ is increasing in its importance due to demand in practical applications such as batteries, capacitors, and display devices, and such processes as electrolytic refining, synthesis and polymerization. The applicability of ionic liquids and supercritical fluids further expands this rich field. Compared with relevant studies on water however, fundamental studies on the molecular and electronic structure of nonaqueous solutions at the electrode-solution interface are left behind these applied studies. This paper aims to approach such fundamental aspects of nonaqueous solutions using nonlinear optical investigation.

\section{Experimental}

Fig. 1 shows the molecular structures of the polar solvents studied. Three representative protonic solvents, water $\left(\mathrm{H}_{2} \mathrm{O}\right)$, methanol $(\mathrm{MeOH})$, and ethanol $(\mathrm{EtOH})$, and one aprotonic solvent, dimethyl sulfoxide (DMSO), were studied. As electrodes, indium-tin-oxide (ITO, $\mathrm{In}_{2} \mathrm{O}_{3}$ doped with $\mathrm{SnO}_{2}$ ) thin films on glass substrates (Geomatec) were used. ITO is an n-type semiconductor and is transparent in the visible region. Its thickness, resistivity, and carrier density are $330 \mathrm{~nm}, 1.2 \times 10^{-4}$ $\Omega \mathrm{cm}$, and $1.0 \times 10^{21} \mathrm{~cm}^{-3}$, respectively.

Measurements were carried out using electromodulation spectroscopy. Fig. 2 shows a schematic of the experimental setup. Detailed descriptions of the experimental method are given in ref. 4, 5 and 16. Two ITO electrodes were immersed in $0.1 \mathrm{M} \mathrm{NaCl}$ aqueous solution or $0.1 \mathrm{M} \mathrm{LiCl}$ organic solution. In order for light to be transmitted through only one electrode, two ITO electrodes of about $5 \mathrm{~mm} \times 10 \mathrm{~mm}$ were mutually perpendicularly oriented to be overlapped at their ends with the ITO surfaces facing each other and separated by a $0.4 \mathrm{~cm}$ thick

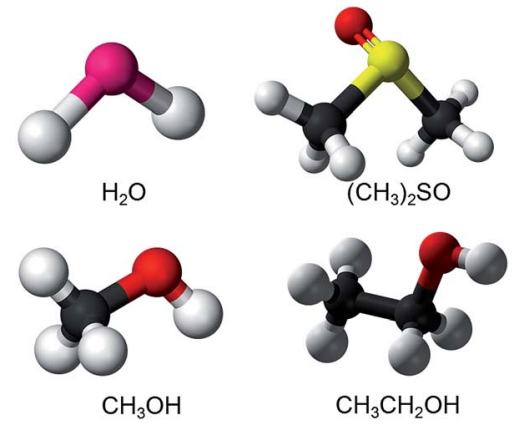

Fig. 1 The molecular structures of the polar solvents studied: water $\left(\mathrm{H}_{2} \mathrm{O}\right)$, dimethyl sulfoxide (DMSO, $\left.\left(\mathrm{CH}_{3}\right)_{2} \mathrm{SO}\right)$, methanol $(\mathrm{MeOH}$, $\left.\mathrm{CH}_{3} \mathrm{OH}\right)$, and ethanol (EtOH, $\left.\mathrm{C}_{2} \mathrm{H}_{5} \mathrm{OH}\right)$.

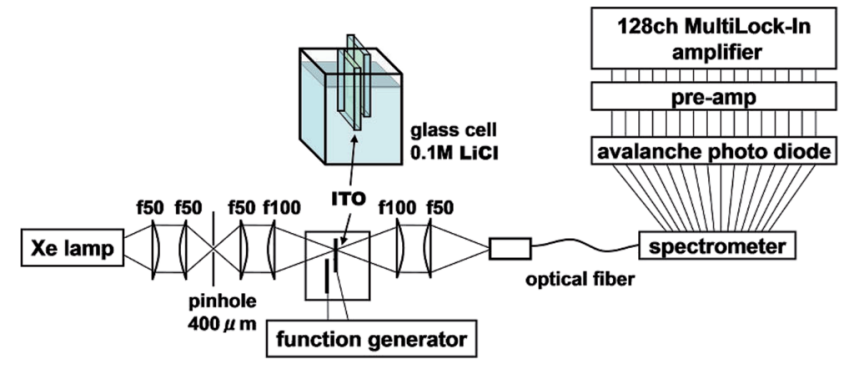

Fig. 2 Experimental setup.

glass spacer. An AC voltage of $2 \mathrm{~V}$ (peak amplitude, $4 \mathrm{~V}_{\mathrm{p}-\mathrm{p}}$ ) was applied between the electrodes at a frequency $f=20 \mathrm{~Hz}$, then the optical constants of the solution changed in the interface between the solution and the electrodes, i.e., in the EDL of the solution and in the space charge layer (SCL) on the surface of the ITO. The probe light from a Xe lamp, whose spatial coherence was enhanced with a pinhole of $0.4 \mathrm{~mm}$, was transmitted normally through one electrode placed at a loosely focused beam position of $c a .3 \mathrm{~mm}$ in diameter. The other ground electrode should show the same size signal with a reversed sign due to the inverse applied voltage. If the probe light is transmitted through both electrodes, the signals cancel resulting in a null signal except in the case of some imbalance between either the geometries of the two electrodes or the sizes of the positive and negative bias signals. The electric-field-induced change in the transmittance was modulated at the AC frequency of $f$ to be detected with a 128-channel lock-in amplifier. The transmitted probe light was focused onto a cross-sectional diameter of $0.5 \mathrm{~mm}$ on the input end of a multimode-fiber bundle connected to a monochromator. For collimating and focusing, quartz lenses with transmittance $>90 \%$ in the $300-1000 \mathrm{~nm}$ range were used. All of the measurements were performed at room temperature. Since $\mathrm{NaCl}$ and $\mathrm{KCl}$ are insoluble in organic solvents, $\mathrm{LiCl}$ is used as an electrolyte for all of the solvents except for water. Differences

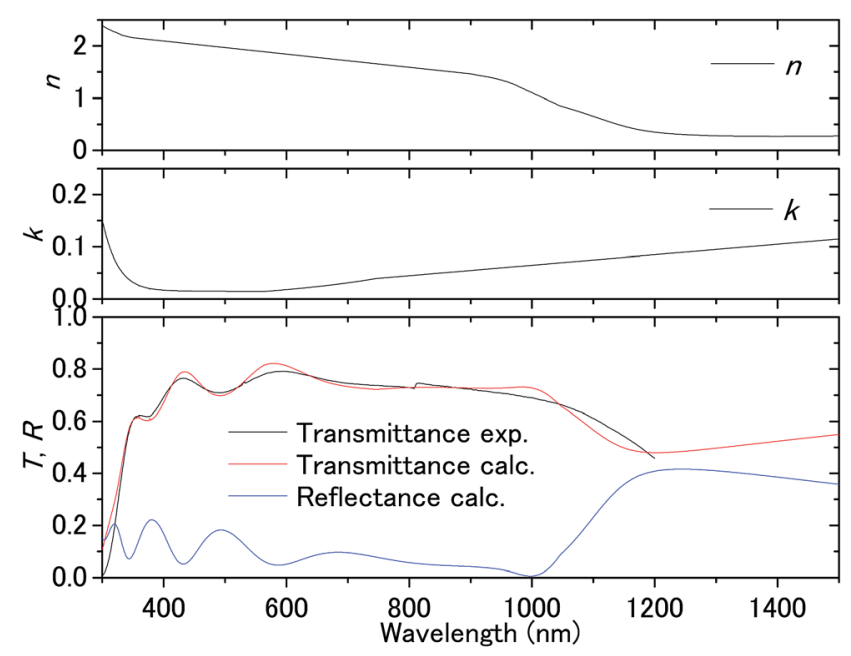

Fig. 3 Transmittance of the ITO substrate in air and the complex refractive index fitted to the experimental transmittance. 


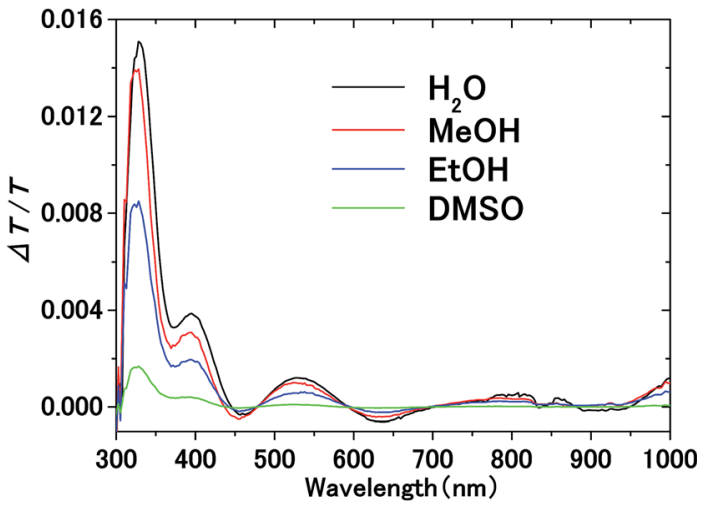

Fig. 4 Electromodulation spectra of solvents on the ITO electrode. AC voltage at frequency $f$ is applied between the electrodes in electrolyte solution.

of the effects among the electrolytes are described in the Appendix.

For impedance measurements of the solution-electrode system, a potentiostat with an impedance analyzer (Model1260A, Solartron) and a galvanostat (Model-1287A, Solartron) were used to make a Cole-Cole plot for the system under experimental conditions as close to those in the electromodulation measurements as possible.

\section{Results}

Fig. 3 shows the transmission spectrum of the ITO electrodes in air. The interference fringes are caused by the $330 \mathrm{~nm}$-thick ITO layer. The decrease in the transmissivity in the UV region is due to absorption above the bandgap energy of ITO. Fig. 4 shows the normalized difference transmission spectrum $\Delta T / T$ at normal incidence. The AC voltage and the modulation frequency were $2 \mathrm{~V}$ (peak amplitude) and $20 \mathrm{~Hz}$, respectively. There is a sharp increase in $\Delta T / T$ in the UV region, while there are interference fringes in the pattern of $\Delta T / T$ from the visible to near-IR regions.

\section{Analyses}

\section{A. Origin of difference transmittance}

The transmittance change in the visible to near-IR region is considered to be due to the change in the refractive index of the solution. However, there is also a possible contribution from the refractive index change of the ITO due to the band population effect. ${ }^{25,26}$ Fig. 5 shows the band structure of an n-type semiconductor. The band structure near the interface bends to form a SCL with the voltage applied. As a result, the absorption edge shifts to the blue (red) side for the positive (negative) bias, leading to a sharp decrease in the absorbance in the UV region. This phenomenon explains the sharp transmission increase in the UV region. The resulting change in the complex refractive index $n_{\mathrm{c}}$ in the SCL of ITO was evaluated using the Lorentz model, where the imaginary part of $\Delta n_{\mathrm{c}}$ was determined to reproduce the observed net difference transmission change in the UV. In the Lorentz model, the real and imaginary parts of $\Delta n_{\mathrm{c}}$ are related through the Kramers-Kronig relations. Fig. 6 shows the assumed complex refractive index change in the SCL in the ITO and the refractive index change in the EDL of the electrolyte solution.

The difference transmittance was then calculated using the transfer matrix method. ${ }^{27,28}$ The characteristic matrix of each layer was calculated with the optical constants of each layer, and that of all of the layers was readily obtained from the product of them. The solution-electrode interface was assumed to be a multilayer system with a constant refractive index for each layer, as shown in Fig. 7. The system structure before voltage application was modeled with a three-layer composition of solution, ITO layer, and substrate. After the voltage was applied, it was modelled with a five-layer structure composed of bulk solution, EDL, SCL, bulk ITO layer, and substrate. As mentioned above, it was assumed that the refractive index change is uniform in each layer. The red solid curves in Fig. 6 show the results calculated with the complex refractive index change in the SCL of ITO and the refractive index change $\Delta n_{\mathrm{s}}$ in the EDL of

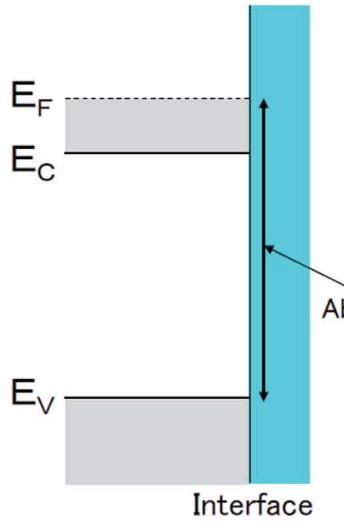

(a)

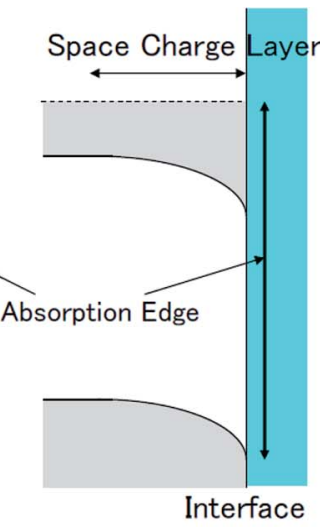

(b)

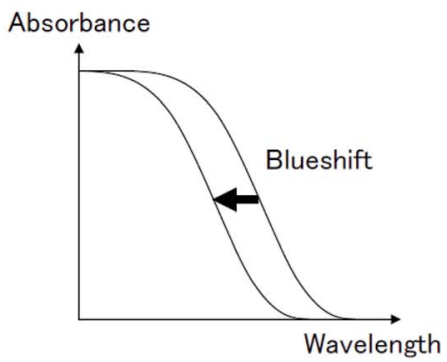

(c)

Fig. 5 The change in the band structure near the surface in an n-type semiconductor caused by the band population effect (a) before and (b) after the application of a positive voltage to the surface, resulting in (c) the blueshift of the absorption edge. $E_{\mathrm{C}}, E_{\mathrm{v}}$, and $E_{\mathrm{F}}$ are the lowest energy in the conduction band, the highest energy in the valence band, and the Fermi energy, respectively. 

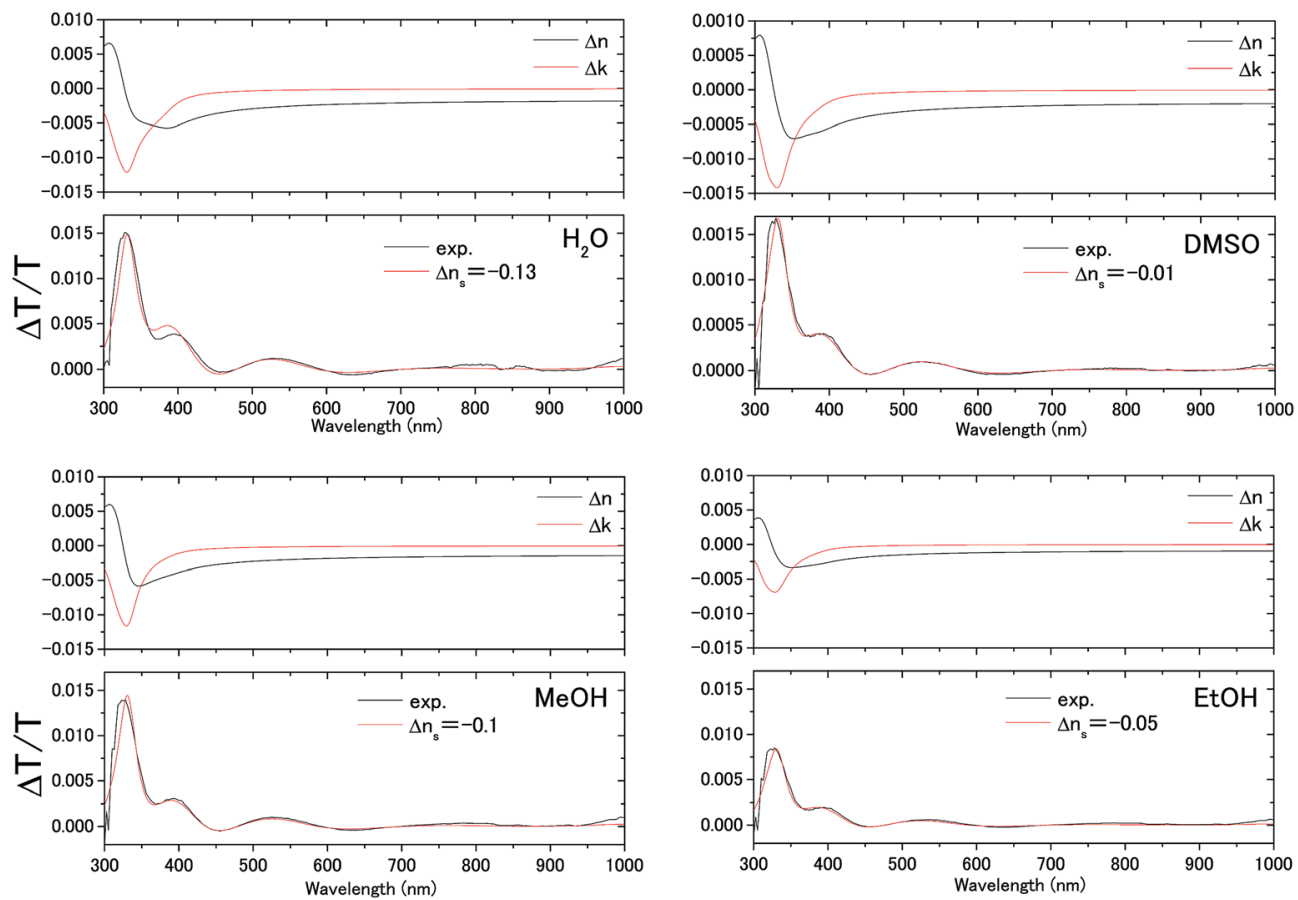

Fig. 6 Measured electromodulation spectra (black lines) and fitted spectra (red lines) with the complex refractive index change $\Delta n+i \Delta k$ in the space charge layer of the ITO electrode shown above and the constant refractive index change $\Delta n_{s}$ in the electric double layer of the solution.

the solutions. $\Delta n_{\mathrm{s}}$ for $\mathrm{H}_{2} \mathrm{O}, \mathrm{MeOH}$, EtOH, and DMSO is -0.13 , $-0.1,-0.05$, and -0.01 , respectively.

\section{B. Voltage drop at interface}

To determine the Pockels constant, the electric field in the EDL should be estimated. The voltage drops in the interfacial layers were analyzed using the impedance measurements. The geometrical positions of the electrodes in the cell and the concentration of LiCl were the same as those in Fig. 2. The impedance of the solution-electrode system is calculated using the formula:
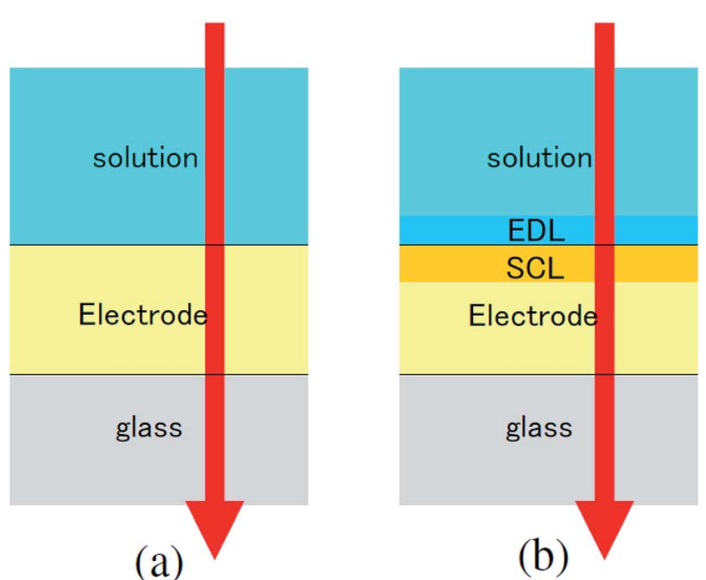

Fig. 7 Model of the interfacial layer: (a) three-layer and (b) five-layer structure before and after the voltage was applied, respectively.

$$
Z^{\prime}=\frac{X}{X^{2}+Y^{2}} E
$$

and

$$
Z^{\prime \prime}=\frac{-Y}{X^{2}+Y^{2}} E,
$$

where $E, X$ and $Y$ are the externally applied AC voltage, and the real and imaginary parts of the signal current in the impedance analyzer, respectively. Fig. 8 shows an equivalent circuit for the interfacial layers, bulk ITO, and bulk solution. It was assumed to be composed of a serial circuit of the solution (resistance) and the thin layers of the SCL and EDL (parallel circuit of capacitance and resistance for each layer). A constant phase element (CPE) was used to fit the impedance of the circuit instead of a capacitance since an RC circuit often cannot be fit

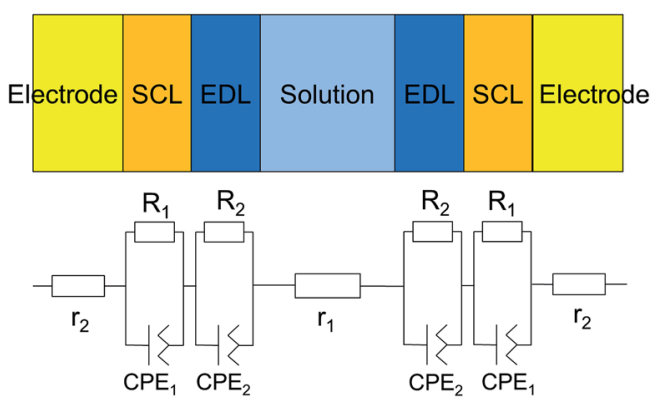

Fig. 8 Equivalent circuit for the measured system, which consists of interfacial layers, the bulk electrode, and the bulk solution. Since the two electrodes are equivalent, the circuit parameters are the same. 

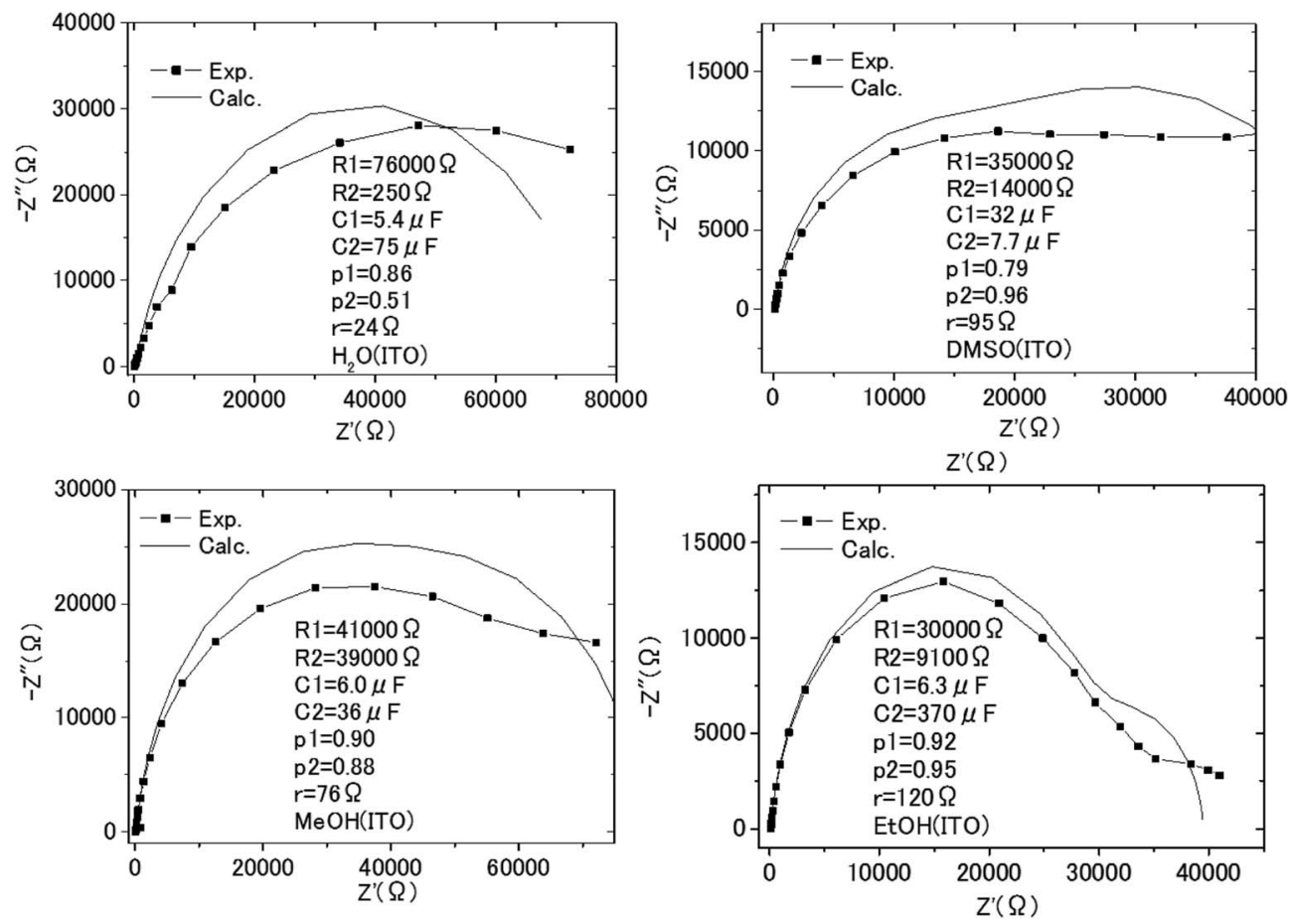

Fig. 9 The Cole-Cole plots obtained with the impedance measurements of the solution-electrode systems.

by a simple capacitance because of the surface roughness. ${ }^{29}$ Impedance of the CPE is expressed as below.

$$
Z_{\mathrm{CPE}}=\frac{1}{C(j \omega)^{p}}
$$

with $C$ as capacitance, $j=\sqrt{-1}, \omega=2 \pi f$ where $f$ is the frequency of the applied AC voltage, and $p$ is a parameter for the surface roughness. Then, the impedance of the equivalent circuit is given by

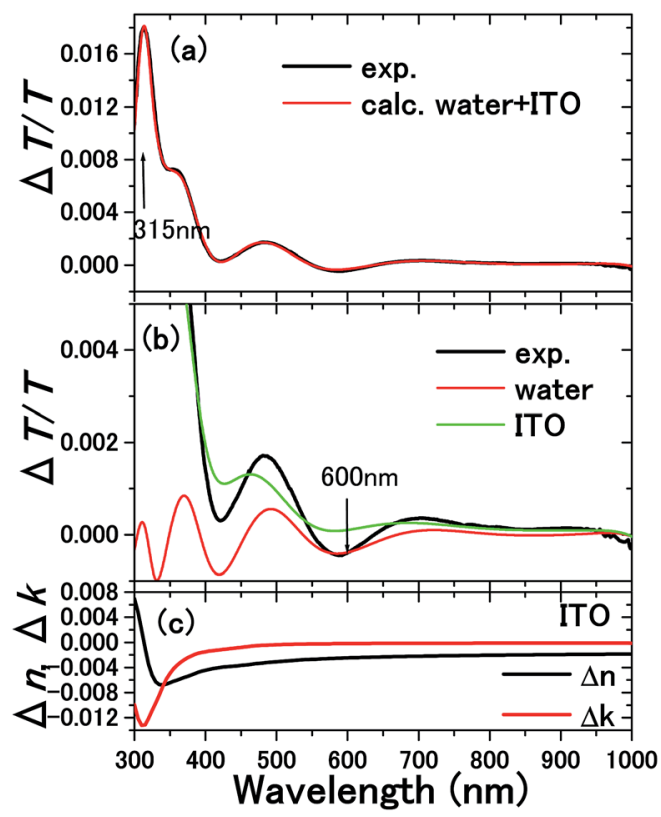

(d)

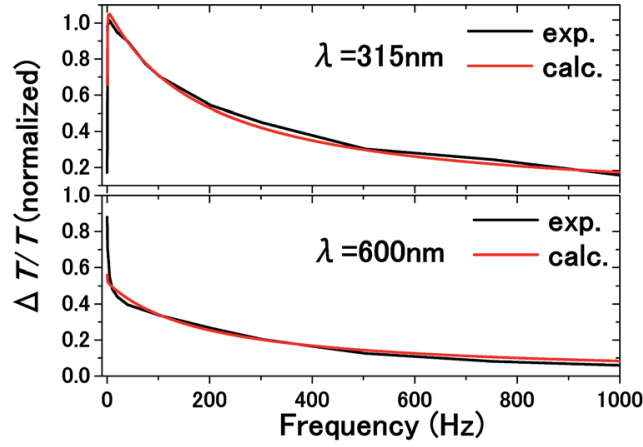

Fig. 10 The experimental and calculated results for $0.1 \mathrm{M} \mathrm{NaCl}$ aqueous solution with a $300 \mathrm{~nm}$ thick ITO electrode are shown in (a). ${ }^{5}$ From the calculations in (b), it is known that the $\Delta T / T$ signals at 315 and $600 \mathrm{~nm}$ exclusively originated from the complex refractive index changes $\Delta n_{\mathrm{c}}=\Delta n+$ $i \Delta k$ in the SCL and EDL, respectively. $\Delta n_{\mathrm{c}}$ assumed for the SCL in ITO is shown in (c). In order to determine the voltage applied to the EDL and SCL, the impedance of each layer was experimentally determined by the frequency dependence of the signals as shown in (d). The signal intensity decreases with frequency because of the impedances involved in the equivalent circuit. Thus, the impedances of the SCL and EDL were independently determined by different frequency dependence of the signals at 315 and $600 \mathrm{~nm}$ to derive the voltage drops of $V_{\mathrm{EDL}}=0.85 \mathrm{~V}$ and $V_{\mathrm{SCL}}=0.15 \mathrm{~V}$. 
Table 1 Voltage division ratios for the solution-electrode systems

\begin{tabular}{llll}
\hline Solvent & EDL & SCL & $r$ \\
\hline $\mathrm{H}_{2} \mathrm{O}$ & 0.92 & 0.07 & 0.01 \\
$\mathrm{MeOH}$ & 0.82 & 0.15 & 0.03 \\
EtOH & 0.93 & 0.01 & 0.06 \\
DMSO & 0.61 & 0.34 & 0.05
\end{tabular}

$$
\begin{aligned}
& Z^{\prime}=\operatorname{Re}\left[\frac{R_{1}}{1+C_{1} R_{1}(j \omega)^{p_{1}}}+\frac{R_{1}}{1+C_{2} R_{2}(j \omega)^{p_{2}}}+r\right] . \\
& Z^{\prime \prime}=\operatorname{Im}\left[\frac{R_{1}}{1+C_{1} R_{1}(j \omega)^{p_{1}}}+\frac{R_{1}}{1+C_{2} R_{2}(j \omega)^{p_{2}}}+r\right] .
\end{aligned}
$$

with $R_{1}, C_{1}$ and $p_{1}$ being the resistance, the capacitance, and the surface roughness parameter in the parallel circuit for the SCL, $R_{2}, C_{2}$, and $p_{2}$ being those for the EDL, and $r_{1}$ and $r_{2}$ are the resistance of the solution and the electrode, respectively, with $r=r_{1}+2 r_{2}$. The impedance of each layer was estimated from the Cole-Cole plot of the frequency dependence of the impedance. The dotted and solid curves in Fig. 9 show the experimental and calculated Cole-Cole plots, respectively. The fit is very good for all of the cases, such that circuit parameters were reliably determined. One problem is that the equivalent circuit is symmetric with respect to the exchange of $R_{1}$ and $C_{1}$ and $R_{2}$ and $C_{2}$ in Fig. 8. Therefore one cannot determine which are for the SCL and for the EDL. Previously, it was carefully analyzed that the applied voltage falls in the interfacial layers with a ratio of EDL : SCL $=0.85: 0.15$ by measuring the frequency dependence of the $\Delta T / T$ signal which is contributed to solely from the SCL in ITO and from the EDL in water as shown in Fig. $10 .^{5}$ Following this result, the voltage drop is concluded to be larger for the EDL than for the SCL. The ratios of the applied voltage at $20 \mathrm{~Hz}$ are summarized in Table 1.

\section{Derivation of Pockels constants}

The Pockels constants were determined from the refractive index change and the electric field in the EDL. In fact, the refractive index change is considered to be anisotropic as represented by the refractive index ellipsoid ${ }^{4,5}$ such that

$$
\left(\frac{1}{n_{\mathrm{w}}^{2}}+r_{13} F_{z}\right)\left(x^{2}+y^{2}\right)+\left(\frac{1}{n_{\mathrm{w}}{ }^{2}}+r_{33} F_{z}\right) z^{2}=1
$$

with $n_{\mathrm{o}}=n_{\mathrm{w}}-\frac{1}{2} n_{\mathrm{w}}{ }^{3} r_{13} F_{z}$ and $n_{\mathrm{e}}=n_{\mathrm{w}}-\frac{1}{2} n_{\mathrm{w}}{ }^{3} r_{33} F_{z}$, where $n_{\mathrm{o}}$ is the refractive index of the ordinary ray (the optical electric field

Table 2 The Pockels coefficients of solvents

\begin{tabular}{ll}
\hline Solvent & $r_{13}\left(\mathrm{pm} \mathrm{V}^{-1}\right)$ \\
\hline $\mathrm{H}_{2} \mathrm{O}$ & 230 \\
$\mathrm{MeOH}$ & 200 \\
EtOH & 84 \\
DMSO & 20
\end{tabular}

$E$ is parallel to the electrode surface while the external electric field $F$ is normal to the surface), $n_{\mathrm{e}}$ is the refractive index of the extraordinary ray (both $E$ and $F$ are normal to the surface), $n_{\mathrm{w}}$ is the refractive index of the bulk solution, and $r_{13}$ and $r_{33}$ are the Pockels constants for the ordinary and extraordinary rays. The refractive index change of solution was assumed to be wavelength independent. In this study, the measurement was performed only at the normal incidence, so that the Pockels constant of solution in the EDL was determined for the ordinary ray, $r_{13}$, only. The results are listed in Table 2. The value for $\mathrm{H}_{2} \mathrm{O}$ agrees well with the previous report. ${ }^{5}$ Note that the values for $\mathrm{H}_{2} \mathrm{O}, \mathrm{MeOH}$, and $\mathrm{EtOH}$ are an order of magnitude larger than those for the typical electro-optic crystal $\mathrm{LiNbO}_{3}: r_{33}=30.8 \mathrm{pm}$ $\mathrm{V}^{-1}$ and $r_{13}=8.6 \mathrm{pm} \mathrm{V}^{-1}{ }^{1}$

\section{Discussion}

It is remarkable that $\mathrm{MeOH}$ and EtOH have as large Pockels constants as $\mathrm{H}_{2} \mathrm{O}$. These solvents are distinguished from DMSO in that they are protonic solvents. This fact strongly suggests that the hydrogen bonding is the most important factor in the Pockels effect in the EDL of these liquids. The Pockels constants are arranged as $\mathrm{H}_{2} \mathrm{O}, \mathrm{MeOH}$, and $\mathrm{EtOH}$ in descending order. This agrees with the order of the strength of the hydrogen bond.

However, more specifically, the following questions arise immediately in terms of the relative magnitudes of the Pockels constants.

Q1. Why does $\mathrm{MeOH}$ have a magnitude very close to that for $\mathrm{H}_{2} \mathrm{O}$ ?

Q2. Why does EtOH have a magnitude less than a half of that for $\mathrm{MeOH}$ ?

Q3. Why does DMSO have a smaller value by one order of magnitude than $\mathrm{H}_{2} \mathrm{O}$ ?

These facts deserve the highest attention, because they should constitute the key factors to the understanding of the microscopic mechanism of the Pockels effect of water. These questions need to be answered in light of the properties of each solvent. For this purpose, the physical properties of the solvents are tabulated in Table $3 .^{30}$

Regarding the strength of the hydrogen bond, if we adopt the difference (increment) in the boiling point in comparison with non-polar molecules with a similar molecular weight, we obtain Table 4.

Notice that DMSO is classified as an aprotonic solvent although it is a protophilic (not protogenic) solvent. Therefore, a large increment in the boiling point is not due to the hydrogen bonding but mainly attributed to its large dipole moment. Therefore, the increment in the b.p. cannot be taken at face value to represent the strength of the hydrogen bond regarding DMSO. Regarding the strength of the hydrogen bond, the hydrogen-bond network, i.e., the average cluster size of molecules due to hydrogen bonding in the pure liquid state, should provide a good measure of the hydrogen-bond strength. The reported cluster size of water ranges from 2 to $280,{ }^{31}$ but there is no recent calculation for the mean cluster size in bulk water. More than two decades ago, the mean cluster size at room temperature was reported to be $50,{ }^{32} 8,{ }^{33}$ and 5 (ref. 34) by the 
Table 3 Physical properties of the solvents at $20^{\circ} \mathrm{C}$

\begin{tabular}{|c|c|c|c|c|c|c|}
\hline Solvent & Molecular weight & $\begin{array}{l}\text { Density (g } \\
\mathrm{cm}^{-3} \text { ) }\end{array}$ & $\begin{array}{l}\text { Dipole moment } \\
\text { (D) }\end{array}$ & $\begin{array}{l}\text { Viscosity } \\
\text { (mPa s) }\end{array}$ & $\begin{array}{l}\text { Static dielectric } \\
\text { constant }\end{array}$ & $\begin{array}{l}\text { Refractive } \\
\text { index }\end{array}$ \\
\hline $\mathrm{H}_{2} \mathrm{O}$ & 18.015 & 0.998 & 1.85 & 1.00 & 80.2 & 1.333 \\
\hline EtOH & 46.07 & 0.789 & 1.44 & 1.2 & 25.3 & 1.361 \\
\hline DMSO & 78.14 & 1.10 & 3.96 & 1.99 & 47.2 & 1.479 \\
\hline
\end{tabular}

Table 4 Increment in the boiling point (b.p.)

\begin{tabular}{llllll}
\hline Solvent & $\begin{array}{l}\text { Increment } \\
\text { in b.p. }\end{array}$ & Molecular weight & Boiling point & Molecule & $\begin{array}{l}\text { Molecular } \\
\text { weight }\end{array}$ \\
\hline $\mathrm{H}_{2} \mathrm{O}$ & 261.5 & 18.015 & 100 & Methane & 16.04 \\
$\mathrm{MeOH}$ & 153.2 & 32.05 & 64.7 & Ethane & 30.07 \\
EtOH & 120.5 & 46.07 & 78.4 & Propane & 44.1 \\
DMSO & 108.9 & 78.14 & 189 & Benzene & 78.11
\end{tabular}

same authors. Regarding $\mathrm{MeOH}$ and $\mathrm{EtOH}$, the average cluster sizes were recently reported independently to be 5.9 for $\mathrm{MeOH}^{35}$ and 4.9 for EtOH. ${ }^{36}$ DMSO is known to exist mainly as a dimer in the pure liquid state ${ }^{37,38}$ due to the dipole-dipole Coulomb attraction (not due to hydrogen bonding). Although these average cluster sizes are not experimentally verified values but dependent on the calculation methods to be updated in future, they are one of the good measures for the hydrogen-bond strength.

$\mathrm{MeOH}$ and $\mathrm{EtOH}$ are similar liquids, thus a difference in the Pockels constant by more than twice is remarkable (Q2). Among the physical properties of them, one cannot find any such physical properties other than viscosity where the magnitude is different by more than twice. Therefore, the viscosity might be one of the important parameters that determines the magnitude of the Pockels effect such that the Pockels constant is negatively correlated with the viscosity value. Note that the voltage division ratio of the DC resistance of the EtOH solution is twice larger than that of the $\mathrm{MeOH}$ solution as shown in Table 1. This is consistent with the viscosity values of the solvents, as the ionic mobility is inversely proportional to the viscosity.

A plausible answer to Q1 is also given by considering not only the hydrogen-bond strength but also the solution viscosity. Both the increment in the b.p. and the average cluster size suggest that the hydrogen-bond strength of $\mathrm{H}_{2} \mathrm{O}$ is about 1.7 times as large as that of $\mathrm{MeOH}$, while the viscosity of $\mathrm{H}_{2} \mathrm{O}$ is also about
1.7 times as large as that of $\mathrm{MeOH}$. If a positive correlation of the hydrogen-bond strength to the Pockels effect is nearly compensated for by a negative correlation of the viscosity, $\mathrm{H}_{2} \mathrm{O}$ and $\mathrm{MeOH}$ should give almost the same magnitude for the Pockels constant, as was observed.

As a bold hypothesis, if we assume that the magnitude of the Pockels constant is proportional to the value of the hydrogenbond strength divided by the viscosity, coincidence between this hypothetical value and the relative magnitude of the Pockels constant is excellent as shown in Table 5.

This is a positive answer to all of the questions: Q1, Q2, and Q3. This coincidence, however, never assures that the physical parameter dependence of the Pockels constant is correctly derived without providing sound physical bases of the microscopic mechanism of the Pockels effect.

It should also be noted that there is an electrode material dependence in the magnitude of the Pockels effect. ${ }^{16}$ The Pockels constant of water in the EDL on the electrode is larger for ITO electrodes than for GaN electrodes. This should be correlated with the fact that interaction of the water molecules with the solid surface by hydrogen bonding is larger for the oxide surface $^{3,39}$ than for the nitride surface.

The present results and the electrode material dependence immediately lead to the following scenario. The biased electrode surface strongly interacts with the solvent molecules by hydrogen bonding. The solvent molecules respond as a cluster ${ }^{39}$

Table 5 Relative magnitude of the Pockels constant compared with (hydrogen-bond strength)/(viscosity)

\begin{tabular}{|c|c|c|c|c|c|c|}
\hline $\mathrm{H}_{2} \mathrm{O}$ & 261.5 & & 1.7 & 1.00 & 1.7 & 1.96 \\
\hline EtOH & 120.5 & 4.9 & 0.78 & 1.2 & 0.65 & 0.71 \\
\hline DMSO & & 2 & 0.32 & 1.99 & 0.16 & 0.17 \\
\hline
\end{tabular}


such that cluster volume and shape are strongly affected by the interaction with the electrode surface as well as by a strong electric field in the electric double layer. This responsivity, i.e., the degree of the change in the volume and shape of the cluster, is larger for a smaller viscosity. As a result, for a larger average cluster size (larger hydrogen-bond strength) and for a smaller viscosity, the refractive index change is larger which occurs asymmetrically being correlated with the volume change or deformation of clusters and being specific to the polarity of the electric field in the EDL. This mechanism is plausible because an $a b$ initio calculation result shows that the dynamic polarizability is proportional to the cluster size of water. ${ }^{\mathbf{4 0}}$

\section{Summary}

The Pockels constants, $r_{13}$, for protonic and aprotonic solvents are evaluated. Protonic solvents, $\mathrm{H}_{2} \mathrm{O}, \mathrm{MeOH}$, and EtOH, have much larger constants than the aprotonic solvent, dimethyl sulfoxide. Water and $\mathrm{MeOH}$ have nearly the same magnitude of Pockels constant, while the Pockels constant of EtOH is less than a half of those of $\mathrm{H}_{2} \mathrm{O}$ and $\mathrm{MeOH}$. The magnitude of the Pockels constants of these four solvents scales with the hydrogen-bond strength (or average cluster size) divided by the solvent viscosity. This result strongly suggests that the strength of the hydrogen bond is the significant source of the Pockels effect. In other words, the magnitude of the Pockels constant could be used as a physical property which characterizes nonaqueous solvents, especially as a good measure of their hydrogen-bond strength.

\section{Appendix}

In the measurement of the Pockels constants, the effect of electrolysis of water should be ruled out. In our experiment, electrolysis occurs when the AC voltage $>6 \mathrm{~V}$ (corresponding to $12 \mathrm{~V}_{\mathrm{p}-\mathrm{p}}$ ) at $20 \mathrm{~Hz}$ is applied to the ITO electrodes immersed in $0.1 \mathrm{M} \mathrm{NaCl}$ aqueous solution. In this case, irreversible change occurs on the ITO surface such that it turns dark in addition to the generation of bubbles. Therefore we carefully avoided electrolysis by setting the AC voltage at $4 \mathrm{~V}_{\mathrm{p}-\mathrm{p}}$, well below the threshold voltage $12 \mathrm{~V}_{\mathrm{p}-\mathrm{p}}$.

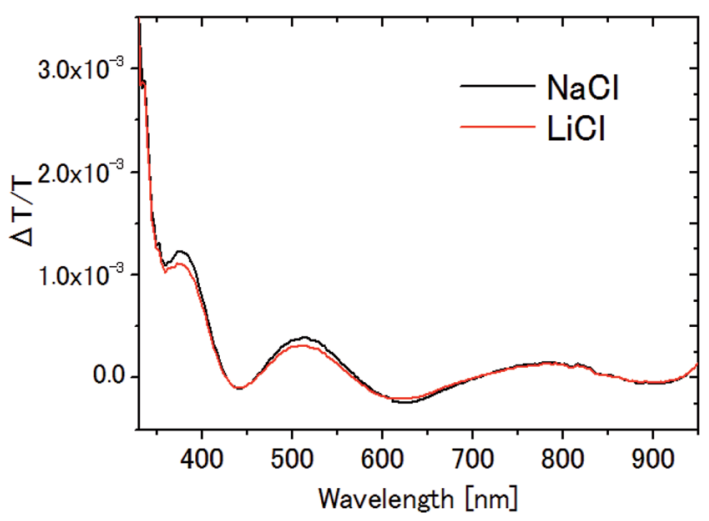

Fig. $11 \Delta T / T$ spectra of $0.1 \mathrm{M} \mathrm{NaCl}$ and $0.1 \mathrm{M} \mathrm{LiCl}$ aqueous solutions.
In this paper, $\mathrm{NaCl}$ is used as an electrolyte for water though $\mathrm{LiCl}$ is used for the other solvents. Fig. 11 shows the $\Delta T / T$ signals for $\mathrm{NaCl}$ aqueous solution and $\mathrm{LiCl}$ aqueous solution. The results agree very well with each other, and are consistent with the previous observation that there was no significant dependence on the kind of electrolyte (between $\mathrm{NaCl}$ and $\mathrm{NaF}^{4}$ ). Even if we take the difference seriously in $\Delta T / T$ between these two aqueous solutions, for example the $6 \%$ difference at the peak at $791 \mathrm{~nm}$, it could affect only the 3 digit-accuracy of the Pockels coefficient.

Since the molar conductivities of aqueous $\mathrm{LiCl}$ and $\mathrm{NaCl}$ solutions are 115.03 and $126.45 \mathrm{~S} \mathrm{~cm}^{2} \mathrm{~mol}^{-1}$ on the infinite dilution condition, ${ }^{\mathbf{4 1}}$ the voltage fall in the bulk solution, which is proportional to $r$ in Table 1, is larger in LiCl solution than in $\mathrm{NaCl}$ solution. As a result, the voltage applied in the EDL should be larger in $\mathrm{NaCl}$ solution than in LiCl solution, yielding a larger signal in NaCl solution than in LiCl solution with the same Pockels constant. The observed small difference is most probably due to this effect.

\section{Conflicts of interest}

There are no conflicts of interest to declare.

\section{Acknowledgements}

This work was supported by a Grant-in-Aid for Scientific Research(C) (Grant Number JP15K05134), and the Japan Society for the Promotion of Science (JSPS).

\section{References}

1 A. Yariv, Quantum Electronics, Wiley, New York, 3rd edn, 1988.

2 D. C. Grahame, Chem. Rev., 1947, 41, 441-501.

3 G. E. Brown Jr, V. E. Henrich, W. H. Casey, D. L. Clark, C. Eggleston, A. Felmy, D. W. Goodman, M. Grätzel, G. Maciel, M. I. McCarthy, K. H. Nealson, D. A. Sverjensky, M. F. Toney and J. M. Zachara, Chem. Rev., 1999, 99, 77-174.

4 E. Tokunaga, Y. Nosaka, M. Hirabayashi and T. Kobayashi, Surf. Sci., 2007, 601, 735-741.

5 Y. Nosaka, M. Hirabayashi, T. Kobayashi and E. Tokunaga, Phys. Rev. B: Condens. Matter Mater. Phys., 2008, 77, 241401.

6 S. Yukita, N. Shiokawa, H. Kanemaru, H. Namiki, T. Kobayashi and E. Tokunaga, Appl. Phys. Lett., 2012, 100, 171108.

7 N. Shiokawa, Y. Mizuno and E. Tokunaga, Opt. Lett., 2012, 37, 2655-2657.

8 Y. Suzuki, K. Osawa, S. Yukita, T. Kobayashi and E. Tokunaga, Appl. Phys. Lett., 2016, 108, 191103.

9 K. Shiratori and A. Morita, J. Chem. Phys., 2011, 134, 234705.

10 D. Rai, A. D. Kulkarni, S. P. Gejji, L. J. Bartolotti and R. K. Pathak, J. Chem. Phys., 2013, 138, 044304.

11 L. Maschio, M. Rérat, B. Kirtman and R. Dovesi, J. Chem. Phys., 2015, 143, 244102. 
12 M. F. Toney, J. N. Howard, J. Richer, G. L. Borges, J. G. Gordon, O. R. Melroy, D. G. Wiesler, D. Yee and L. B. Sorensen, Surf. Sci., 1995, 335, 326-332.

13 Y. S. Chu, T. E. Lister, W. G. Cullen, H. You and Z. Nagy, Phys. Rev. Lett., 2001, 86, 3364-3367.

14 W. F. Murphy, J. Chem. Phys., 1977, 67, 5877-5882.

15 G. Maroulis, J. Chem. Phys., 1991, 94, 1182-1190.

16 H. Kanemaru, Y. Nosaka, A. Hirako, K. Ohkawa, T. Kobayashi and E. Tokunaga, Opt. Rev., 2010, 17, 352-356. 17 M. F. Chaplin, Biophys. Chem., 1999, 83, 211-221.

18 F. N. Keutsch and R. J. Saykally, Proc. Natl. Acad. Sci. U. S. A., 2001, 98, 10533-10540.

19 P. Wernet, D. Nordlund, U. Bergmann, M. Cavalleri, M. Odelius, H. Ogasawara, L. A. Näslund, T. K. Hirsch, L. Ojamäe, P. Glatzel, L. G. M. Pettersson and A. Nilsson, Science, 2004, 304, 995-999.

20 M. Matsumoto, A. Baba and I. Ohmine, J. Chem. Phys., 2007, 127, 134504.

21 I. Bakó, A. Bencsura, K. Hermannson, S. Bálint, T. Grósz, V. Chihaia and J. Oláh, Phys. Chem. Chem. Phys., 2013, 15, 15163-15171.

22 G. C. Pimentel and A. L. McClellan, The hydrogen bond, Freemann, San Francineo, 1960.

23 Organic Electrochemistry, ed. O. Hammerich and B. Speiser, CRC Press, 5th edn, Revised and Expanded, 2015.

24 K. Izutsu, Electrochemistry in Nonaqueous Solutions, Wiley VCH, 2002.

25 N. Bottka, D. L. Jhonson and R. Glosser, Phys. Rev. B: Solid State, 1977, 15, 2184-2194.
26 R. Glosser and B. O. Seraphin, Phys. Rev., 1969, 187, 10211024.

27 M. Born and E. Wolf, Principles of Optics, Pergamon Press, Oxford, 6th edn, 1980.

28 M. Kobiyama, Theory of Optical Thin Films, Optronics, Tokyo, 2nd edn, 2003, p. 83, in Japanese.

29 H. Fricke, Philos. Mag., 1932, 14, 310-318.

30 Handbook of Chemistry: Pure Chemistry, Maruzen, 5th edn, 2004; http://solvdb.ncms.org/; Chronological Scientific Tables, Maruzen, 2016; CRC Handbook of Chemistry and Physics, ed. W. M. Haynes, CRC Press, 92nd edn, 2011.

31 R. Ludwig, Angew. Chem., Int. Ed., 2001, 40, 1808-1827.

32 G. Nemethy and H. A. Scheraga, J. Chem. Phys., 1962, 36, 3382-3400.

33 A. T. Hagler, H. A. Scheraga and G. Nemethy, J. Phys. Chem., 1972, 76, 3229-3243.

34 B. R. Lentz, A. T. Hagler and H. A. Scheraga, J. Phys. Chem., 1974, 76, 1531-1550.

35 P. Sillren, J. Bielecki, J. Mattsson, L. Borjesson and A. Matic, J. Chem. Phys., 2012, 136, 094514.

36 J. Janecek and P. Paricaud, J. Chem. Phys., 2013, 139, 174502.

37 T. Varnali, Struct. Chem., 1996, 7, 111-118.

38 T. Shikata and N. Sugimoto, Phys. Chem. Chem. Phys., 2011, 13, 16542-16547.

39 M. A. Henderson, Surf. Sci. Rep., 2002, 46, 1-308.

40 T. K. Ghanty and S. K. Ghosh, J. Chem. Phys., 2003, 118, 8547-8550.

41 D. A. MacInnes, The Principles of Electrochemistry, Reinhold, New York, 1961. 